\title{
Gradient elasticity modelling for the analysis of fiber composites with fiber resistant to flexure
}

\author{
Mahdi Zeidi, Chun IL Kim \\ Mechanical Engineering Department \\ University of Alberta \\ Edmonton, AB, Canada \\ e-mails: zeidi@ualbert.ca, cikim@ualberta.ca
}

\begin{abstract}
A model for the deformation of an elastic solid reinforced by embedded fibers is presented in which elastic resistance of the fibers to bending is incorporated. We developed the equilibrium equations and necessary boundary conditions, Within the framework of strain-gradient elasticity, which describe the finite plane deformations of fiber-reinforced composite materials. The resulting nonlinear coupled $4^{\text {th }}$ order partial differential equations are numerically solved by using the FEM. Within the limitation of superposed incremental deformations, a complete analytical solution is also obtained.
\end{abstract}

Finite elasticity; strain gradient theory; fiber-reinforced materials; flexure; superposed incremental deformations; finite element method; finite plane deformations

\section{INTRODUCTION}

The mechanics of fiber-reinforced materials is a wellestablished subject [1-4] that has significantly advanced our knowledge on and practices in the development of composite materials. One way of addressing this problem is to examine the local behavior of an individual microstructure-matrix system including the interfacial region. Such investigations are essential to extracting the mechanical properties of those materials, yet rather inefficient at predicting the general behavior of the composite materials under prescribed forces and/or displacements. Within this prescription, much theoretical work has been developed based on the simple concept of an anisotropic material where the material response function depends on the classical deformation gradient with the augmented constraints of bulk incompressibility and fiber inextensibility. A general theory for an elastic solid with fibers resistant to flexure, stretch and twist is presented in [4].

In the present work, we develop a continuum model in which the fibers accommodate elastic resistance to flexure. The fibers are treated as continuously distributed spatial rods of the Kirchhoff type where the kinematics are based on their position field and a director field. We seek a complete model describing the finite plane deformation of fiber composites which offers fiber resistance to flexure loading. The basic kinematics and constitutive framework are presented in Section II. Via the computation of variational derivatives and the virtual-work statement, in Section II the corresponding equilibrium equation

NSERC of Canada via Grant \#RGPIN 04742 and U of A start-up grant. is derived in which the bulk incompressibility condition is augmented in a weak sense. In section III, we also consider an example in the case of Neo-Hookean materials with necessary boundary conditions. In section IV, a set of numerical solutions is obtained via a finite element analysis. In Section $\mathrm{V}$, a complete analytical solution of the linearized system is obtained for the case when the fiber composite is subjected to uniform bending moment at its edge. It is good to mention that the presented model can serve as an alternative 2D Cosserat theory of nonlinear elasticity.

\section{KINEMATICS AND EQUILIBRIUM EQUATIONS}

We propose that the mechanical response of the fiber material is governed by the following strain energy function:

$$
\begin{gathered}
\mathrm{W}(\mathbf{F}, \mathbf{G})=\widehat{W}(\mathbf{F})+\mathrm{W}(\mathbf{G}), \\
\mathrm{W}(\mathbf{G})=\frac{1}{2} \mathrm{C}(\mathbf{F})|\mathrm{g}|^{2} .
\end{gathered}
$$

where $\mathbf{F}$ and $\mathbf{G}$ are the gradient of the deformation and the second gradient of the deformation, respectively. Further, C refers to the material property of fibers which, in general, independent of the deformation gradient. The orientation of a particular fiber is given by

$$
\begin{aligned}
& \lambda=|\mathbf{d}|, \\
& \tau=\lambda^{-1} \mathbf{d} .
\end{aligned}
$$

Where

$$
\mathbf{d}=\mathbf{F D}
$$

in which $\mathbf{D}$ is the unit tangent to the fiber trajectory in the reference configuration. Equation (3) can be derived by taking the derivative of $r(s)=\chi(\mathbf{X}(\mathrm{s}))$, upon making the identifications $\mathrm{D}=\mathrm{X}^{\prime}(\mathrm{s})$ and $\mathrm{d}=r^{\prime}(\mathrm{s})$. The expression for geodesic curvature of an arc (r (s)) is then obtained from (3) as

$$
\mathbf{g}=\mathbf{r}^{\prime \prime}=\frac{\mathrm{d}\left(\mathbf{r}^{\prime}\right)}{\mathrm{ds}}=\frac{\partial(\mathbf{F} . \mathbf{D})}{\partial \mathbf{X}} \frac{\mathrm{d} \mathbf{X}}{\mathrm{ds}}=\nabla|\mathbf{F D}| \mathbf{D}
$$

To accommodate the bulk incompressibility condition, we consider the following energy functional 


$$
\begin{gathered}
\mathrm{E}=\int_{\mathrm{w}} \mathrm{U}(\mathbf{F}, \mathbf{G}, p) \mathrm{dA}, \\
\mathrm{U}(\mathbf{F}, \mathbf{G}, p)=\mathrm{W}(\mathbf{F}, \mathbf{G})-p(J-1) .
\end{gathered}
$$

where $J$ is determinant of $\mathbf{F}$ and $p$ is a Lagrange-multiplier field. The induced variation of the energy is then evaluated as

$$
\dot{\mathrm{E}}=\int_{\mathrm{w}} \dot{\mathrm{W}}(\mathbf{F}, \mathbf{G}) \mathrm{dA}
$$

Where

$$
\dot{U}(\mathbf{F}, \mathbf{G}, p)=W_{\mathbf{F}} \cdot \dot{\mathbf{F}}-W_{\mathbf{G}} \cdot \dot{\mathbf{G}}-p \dot{J}
$$

So we have

$$
P_{i A}=\frac{\partial W}{\partial F_{i A}}-p F_{i A}^{*}-C g_{i, B} D_{B} D_{A}
$$

Therefore, the corresponding Euler equation can be obtained as

$$
P_{i A, A}=0 \text { or } \operatorname{Div}(\mathbf{P})=0
$$

\section{NEO-HOOKEAN MATERIALS}

In the case of incompressible Neo-Hookean materials, the energy density function is given by

$$
\begin{gathered}
\widehat{\mathrm{W}}(\mathbf{F})=\frac{\mu}{2} \operatorname{tr}(\mathbf{C})=\frac{\mu}{2} \operatorname{tr}\left(\mathbf{F}^{\mathrm{T}} \mathbf{F}\right)=\frac{\mu}{2} \mathbf{F} \cdot \mathbf{F} \\
\mathrm{W}(\mathbf{F}, \mathbf{G})=\frac{\mu}{2} \mathbf{F} \cdot \mathbf{F}+\frac{1}{2} \mathrm{C}|\mathrm{g}|^{2}
\end{gathered}
$$

where $\mu$ and $\mathrm{C}$ are the material constant of the matrix and fiber, respectively. Accordingly, the corresponding Euler equation can be obtained as

$$
P_{i A, A}=\mu F_{i A, A}-p_{, A} F_{i A}^{*}-C g_{i, A B} D_{A} D_{B}=0
$$

where $\varepsilon_{\mathrm{ij}}$ is the 2-D permutation; $\varepsilon_{12}=-\varepsilon_{21}=1, \varepsilon_{11}=-\varepsilon_{22}=0$. Equation (12) together with the incompressibility condition furnishes a coupled PDE system for $\chi_{1}, \chi_{2}$ and p. i.e.

$$
\begin{gathered}
\mu\left(\chi_{1,11}+\chi_{1,22}\right)-p_{, 1} \chi_{2,2}+p_{, 2} \chi_{2,1}-C \chi_{1,1111}=0 \\
\mu\left(\chi_{2,11}+\chi_{2,22}\right)+p_{, 1} \chi_{1,2}-p_{, 2} \chi_{1,1}-C \chi_{2,1111}=0 \\
\chi_{1,1} \chi_{2,2}-\chi_{2,1}=1 .
\end{gathered}
$$

When the fiber's directions are either normal or tangential to the boundary, we have

$$
\begin{gathered}
t=P_{i A} N_{A}, \\
m_{i}=C g_{i} D_{A} N_{A} D_{B} N_{B},
\end{gathered}
$$

$$
f_{i}=0 \text {. }
$$

where

$$
\begin{gathered}
P_{i A}=\mu F_{i A}-p F_{i A}^{*}-g_{i, B} D_{A} D_{B}, \\
g_{i}=F_{i A, B} D_{A} D_{B} .
\end{gathered}
$$

\section{FINITE ELEMENT ANALYSIS OF THE 4TH ORDER COUPLED PDE}

It is not trivial to demonstrate numerical analysis procedures for coupled PDE systems, especially for those with high order terms, since the piece wise linear function adopted in FE analysis has limited differentiability up to second order. For preprocessing, Equation (13) can be recast as

$$
\begin{gathered}
\mu\left(\mathrm{R}+\chi_{1,22}\right)-p_{, 1} \chi_{2,2}+p_{, 2} \chi_{2,1}-\mathrm{C} \chi_{1,1111}=0, \\
\mu\left(\mathrm{Q}+\chi_{2,22}\right)+p_{, 1} \chi_{1,2}-p_{, 2} \chi_{1,1}-\mathrm{C} \chi_{2,1111}=0, \\
\mathrm{Q}-\chi_{1,11}=0 \\
\mathrm{R}-\chi_{2,11}=0, \\
\mathrm{~A}-\mu\left(\chi_{1,11}+\chi_{1,22}\right)-\mathrm{CR}_{, 11}=0 \\
\mathrm{~A}-\mu\left(\chi_{2,11}+\chi_{2,22}\right)-\mathrm{CQ}_{, 11}=0 .
\end{gathered}
$$

where $\mathrm{Q}=\chi_{1,11}$ and $\mathrm{R}=\chi_{2,11}$. By employing the Picard iterative process, the nonlinear terms in the above can be treated as

$$
\begin{gathered}
-\mathrm{A}_{\text {initial }} \chi_{2,2}^{\text {initial }}+\mathrm{B}_{\text {initial }} \chi_{2,1}^{\text {initial }} \Longrightarrow-\mathrm{A}_{0} \chi_{2,2}^{0}+\mathrm{B}_{0} \chi_{2,1}^{0} \\
\mathrm{~A}_{\text {initial }} \chi_{1,2}^{\text {initial }}-\mathrm{B}_{\text {initial }} \chi_{1,1}^{\text {initial }} \Longrightarrow \mathrm{A}_{0} \chi_{1,2}^{0}-\mathrm{B}_{0} \chi_{1,1}^{0}
\end{gathered}
$$

Thus, we write

$$
\begin{gathered}
-\mathrm{A}_{\mathrm{N}-1} \chi_{2,2}^{\mathrm{N}-1}+\mathrm{B}_{\mathrm{N}-1} \chi_{2,1}^{\mathrm{N}-1} \Longrightarrow-\mathrm{A}_{\mathrm{N}} \chi_{2,2}^{\mathrm{N}}+\mathrm{B}_{\mathrm{N}} \chi_{2,1}^{\mathrm{N}} \\
\mathrm{A}_{\mathrm{N}-1} \chi_{1,2}^{\mathrm{N}-1}-\mathrm{B}_{\mathrm{N}-1} \chi_{1,1}^{\mathrm{N}-1} \Longrightarrow \mathrm{A}_{\mathrm{N}} \chi_{1,2}^{\mathrm{N}}-\mathrm{B}_{\mathrm{N}} \chi_{1,1}^{\mathrm{N}}
\end{gathered}
$$

where the values of A and B continue to be refreshed based on their previous estimations as iteration progresses. The weak form of Eq. (16)1 is given by

$$
0=\int_{\Omega} \mathrm{w}_{1}\left(\mu\left(\mathrm{R}+\chi_{1,22}\right)-\mathrm{A} \chi_{2,2}+\mathrm{B} \chi_{2,1}-\mathrm{CR}_{, 11}\right) \mathrm{d} \Omega
$$

where $\Omega, \partial \Gamma$ and $\mathrm{N}$ are the domain of interest, the associated boundary, and the rightward unit normal to the boundary $\partial \Gamma$ in the sense of the Green-Stoke's theorem, respectively. The unknowns, $\chi_{1}, \chi_{2}, \mathrm{Q}, \mathrm{R}, \mathrm{A}$ and $\mathrm{B}$ can be written in the form of Lagrangean polynomial. Finally, we assemble the local stiffness matrices and obtain the following systems of equations in the Global form. 
For demonstration purpose, we consider a rectangular fiber composite where one end is fixed and the other end is subjected to uniform bending in order to examine fibers' reinforcing effects against to flexure. We also note here that data are obtained under the normalized setting. The convergence criteria are set for both nonlinear terms (i.e. A and B) and the deformed profiles at $\mathrm{y}=0$.

$$
\begin{aligned}
& \left|A_{n+1}-A_{n}\right|=e_{1} \leq \varepsilon, \\
& \left|B_{n+1}-B_{n}\right|=e_{2} \leq \varepsilon,
\end{aligned}
$$$$
\varepsilon=\text { Maximum error. }
$$

It is clear from Fig. 1 that the adopted numerical method demonstrates fast convergence within 20 iterations. The deformation profile and contour show smooth transitions as they approach the boundary (Figs. 2, 3).

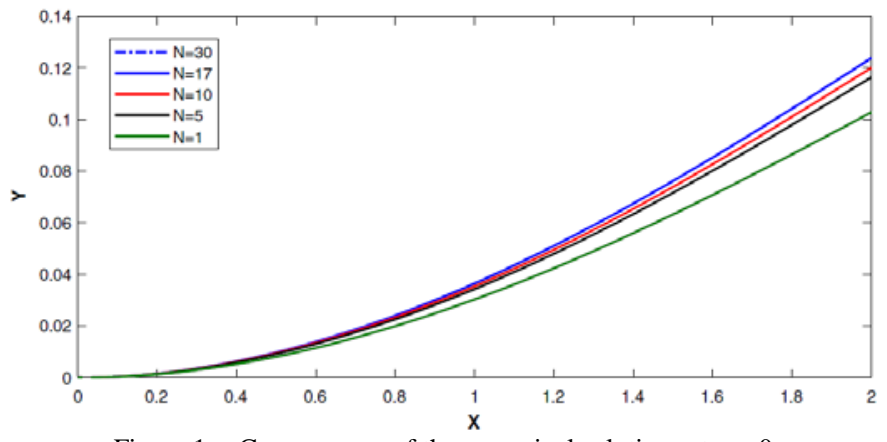

Figure 1. Convergence of the numerical solutions at $y=0$

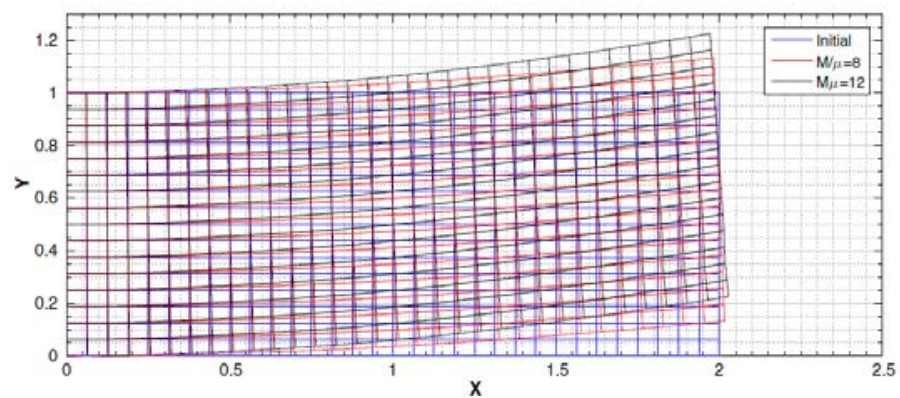

Figure 2. Deformed configurations with respect to $M / \mu$ when $C / \mu=150$

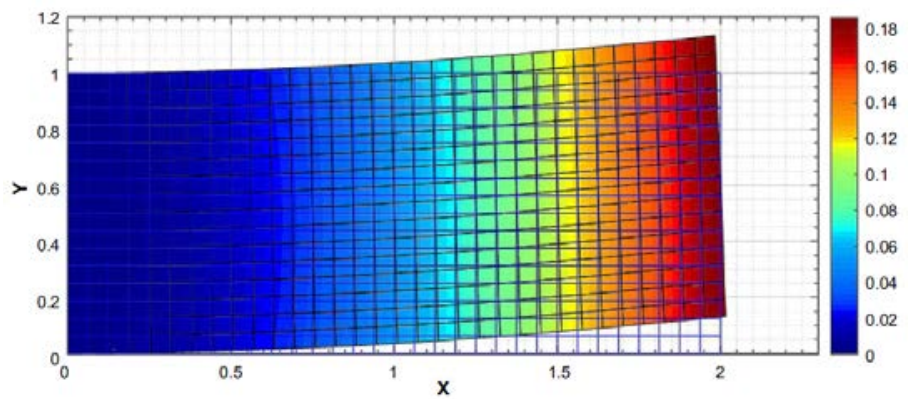

Figure 3. Deformation contour when $\mathrm{C} / \mu=150$ and $\mathrm{M} / \mu=8$

\section{SOLUTION TO THE LINEARIZED PROBLEM}

We introduce scalar field $\phi$ as

$$
\begin{gathered}
\mathbf{u}=\mathbf{k} \times \nabla \phi, \mathbf{k}(\text { unit normal), } \\
u_{i}=\varepsilon_{\lambda i} \phi_{, \lambda},
\end{gathered}
$$

The linearized Euler equation can be rewritten as

$$
\dot{P}_{, i}=\mu \varepsilon_{\lambda i}\left(\phi_{, \lambda 11}+\phi_{, \lambda 22}\right)-C \varepsilon_{\lambda i} \phi_{, \lambda 1111}
$$

So the final equation will be

$$
\begin{gathered}
\Delta H-\alpha H_{, 1111}=0, \\
H=\Delta \phi \text { and } \alpha=\frac{C}{\mu}>0 .
\end{gathered}
$$

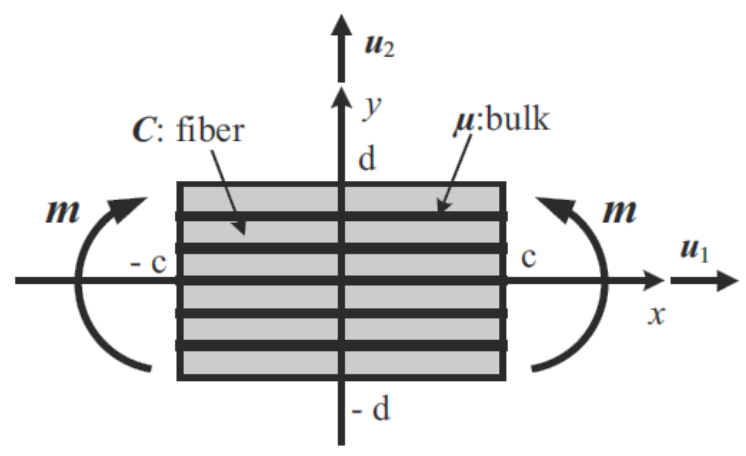

Figure 4. Schematic of problem

The general solution for the above equation can be found as

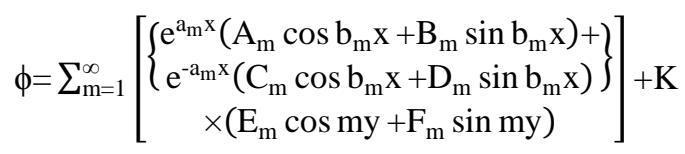

where $\mathrm{K}$ is a solution of Laplace's equation given by

$$
K=\sum_{n=1}^{\infty}\left[\left(G_{n} \cosh n x+H_{n} \sinh n x\right)\left(I_{n} \cos n y+J_{n} \sin n y\right)\right]
$$

In above equations $\mathrm{a}_{\mathrm{m}}$ and $\mathrm{b}_{\mathrm{m}}$ are

$$
a_{m}=\frac{\sqrt{2 m \sqrt{\alpha}+1}}{2 \sqrt{\alpha}}, b_{m}=\frac{\sqrt{2 m \sqrt{\alpha}-1}}{2 \sqrt{\alpha}} .
$$

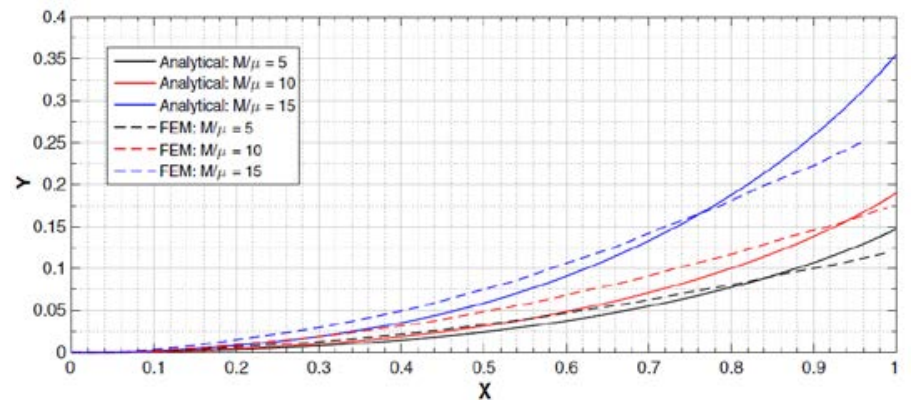


Figure 5. Solutions of the bending problem with $C / \mu=150$. Nonlinear solution (dashed line), linear solution (solid line)

The analytical (linear) solution shows good agreement with nonlinear solution (FEM) for the small deformation regime, while larger values of $\mathrm{M}$ induce a significant discrepancy between the linear and nonlinear solution (see, Fig. 5). As a result, the obtained analytical solution has limitations in large deformation analysis. However, it can still be used in the design and analysis of fiber composites, particularly for CNCreinforced composites, where the deformations of the systems are expected to be relatively 'small'.

\section{CONCLUSION}

A continuum-based model is developed in finite plane elastostatics in which fibers resistant to flexure is taken into account. The fibers are regarded as continuously distributed spatial rods of the Kirchhoff type in which the kinematics are based on a position field and a director field. The equilibrium equation of the fiber-reinforced composite materials is derived by the variational computation and method of virtual work.

These constitute a highly nonlinear coupled PDE system which is treated numerically via the FEM. Within the prescription of superposed incremental deformations, a complete linear theory is developed through which an analytical solution of the corresponding linearized PDE system is obtained. The obtained analytical solution demonstrates good agreement with nonlinear solution for the small deformation regime, yet has limited predictions for large deformation analysis.
The author would like to thank Dr. Steigmann for stimulating his interest in this subject. This work was supported by the Natural Sciences and Engineering Research Council of Canada via Grant \#RGPIN 04742 and the University of Alberta through a start-up grant.

\section{REFERENCES}

[1] J.F. Mulhern, T.G. Rogers, A.J.M. Spencer, "A continuum theory of a plastic-elastic fibre-reinforced material,” Int. J. Eng. Sci., 1969, vol. 7, pp. 129-152

[2] A.C. Pipkin, T.G Rogers, "Plane deformations of incompressible fiberreinforced materials,” ASME J. Appl. Mech., 1971, vol. 38(8), pp. 634640.

[3] A.J.M. Spencer, K.P. Soldatos, "Finite deformations of fibre-reinforced elastic solids with fibre bending stiffness,” Int. J. Nonlinear Mech., 2007, vol. 42, pp. 355-368.

[4] P. Neff, "A finite-strain elastic-plastic Cosserat theory for polycrystals with grain rotations,” Int. J. Eng. Sci., 2006, 44, pp. 574-594.

[5] D.J. Steigmann, "Theory of elastic solids reinforced with fibers resistant to extension, flexure and twist,” Int. J. Nonlinear Mech., 2012, vol. 47(7), pp. 734-742.

[6] M. Zeidi, C.I. Kim, "Mechanics of fiber composites with fibers resistant to extension and flexure," Math. Mech. Solids, 2017, doi.org/10.1177/1081286517728543.

[7] F. dell'Isola, D.J Steigmann, "A two-dimensional gradient-elasticity theory for woven fabrics,” J. Elast., 2015, vol. 118(1), pp. 113-125.

[8] C. Truesdell., W. Noll, "The non-linear field theories of mechanics. In: Flugge, S. (ed.) Handbuch der Physik, vol. III/3,” Springer, Berlin, 1965.

[9] M. Zeidi, C.I. Kim, "Finite plane deformations of elastic solids reinforced with fibers resistant to flexure: complete solution," Arch. Appl. Mech., 2018, doi.org/10.1007/s00419-018-1344-3.

[10] N.F. Dow, "Study of stresses near a discontinuity in a filament-reinforced composite material,” Gen Elect Co Report number R63-SD-61, 1963. 